\title{
Optimized Plasmid Construction Strategy for Cas9
}

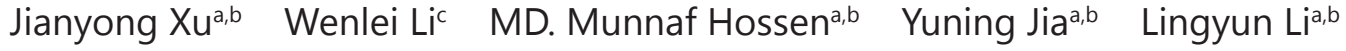 \\ Zhong Huanga,b \\ aDepartment of Immunology, School of Medicine, Shenzhen University, Shenzhen, 'Institute of \\ Biological Therapy, Shenzhen University, Shenzhen, 'Department of Obstetrics, Women and Children \\ Health Institute of Futian, Shenzhen, P.R. China
}

\section{Key Words}

RNA guided endonuclease • Genome editing • Cas9 • Guide RNA • gRNA

\begin{abstract}
Background/Aims: The target genome editing technology not only plays an important role in basic biology studies but also holds a great promise for potential clinical applications. The new generation of engineered nuclease RGEN (RNA Guided EndoNuclease) is much easier to construct and modify, and attracts more attentions. In the current study, we compared different plasmid construction strategies of Cas9-gRNA (guide RNA). Methods: Different plasmid construction strategies of Cas9-gRNA were compared. And more modifications were introduced into the plasmid construction strategy. Results: The plasmid construction efficiency of expressing the gRNA and Cas9 in one plasmid was lower than expressing them in two separate plasmids. However, they showed the similar genome editing efficiency. We further introduced the Golden-gate assembly and blue-white screening approaches into the Cas9gRNA construction procedures, without the process of vector digestion and gel purification. Conclusions: Combing with the optimized gRNA structure (gRNA-BL) we identified before, we established one more cost-effective, time-saving and efficient plasmid construction strategy for Cas9-gRNA.
\end{abstract}

\section{Introduction}

Since the first demonstration of the genome editing technique, this technology has been studied intensively and developed rapidly. Now we could manipulate the genome specifically and precisely in different ways, such as knock-out (deleting a fragment of DNA sequence), knock-in (introducing extra DNA fragment) and target gene correction/mutation (DNA sequence alterations) [1-4]. Genome editing has been widely applied in different organisms for both basic studies and also therapeutic applications [1-4].

\begin{tabular}{ll}
\hline Jianyong Xu & Department of Immunology, School of Medicine, Shenzhen University \\
& Nanhai Avenue 3688, Shenzhen 518060, Guangdong, (China) \\
& Tel. +86 13641473906, E-Mail xujianyong@szu.edu.cn
\end{tabular}


Differing from the first generation of genome editing techniques, including ZFN (Zinc Finger Nucleases) and Talen (Transcription Activator-Like Effector Nucleases), the second generation of genome editing tool RGEN (RNA Guided EndoNuclease) is much easier to construct and modify, and more efficient, attracting more attentions [1, 5-7]. However, more efforts should be made to improve both the efficiency and the specificity of the RGEN[8]. Previously, we had identified one optimized guide RNA structure with higher genome editing efficiency [9]. Then we wanted to establish a cost-effective and time-saving plasmid construction platform for high throughput genome editing. Thus we compared different plasmid construction strategies here. After introducing more modifications, we established one more cost-effective, time-saving and efficient plasmid construction strategy for Cas9gRNA.

\section{Materials and Methods}

\section{Plasmid Construction}

RGEN plasmids were obtained from Addgene, including FZ (Addgene 42229, pX260-U6-DR-BB-DRCbh-NLS-hSpCas9-NLS-H1-shorttracr-PGK-puro)[10], cFZ (Addgene 42230, pX330-U6-Chimeric_BB-CBhhSpCas9)[10], FZM (Addgene 42333, pX334-U6-DR-BB-DR-Cbh-NLS-hSpCas9n(D10A)-NLS-H1-shorttracrPGK-puro)[10], cFZM (Addgene 42335, pX335-U6-Chimeric_BB-CBh-hSpCas9n(D10A))[10], GMC (Addgene 41824, gRNACloning Vector)[11], JKJ (Addgene 43860, MLM3636), hCas9 (Addgene 41815, hCas9)[11]. Plasmid DR-gRNA and BL (gRNA-BL) were constructed as described previously [9]. The guide RNA oligos targeting human gene Desmin were designed and constructed as described previously [9].

RGEN plasmids were digested with corresponding restriction enzymes (BbsI for FZ, FZM, cFZ, cFZM, DR-gRNA; AflII for GMC; and BsmBI for JKJ and BL. All restriction enzymes were purchased from New England Biolabs), gel purified and used for ligation. $1 \mu \mathrm{L}$ annealed oligos were mixed with $0.5 \mu \mathrm{L}$ Quick T4 DNA Ligase, $5 \mu \mathrm{L} 2 \mathrm{X}$ Quick Ligation Buffer (New England Biolabs) and corresponding vectors (50ng FZ and FZM, 10kb; 42ng cFZ and cFZM, 8.4kb; 13ng DR-gRNA, 2.6kb; 11ng JKJ and BL, 2.2kb) in a total volume of $10 \mu \mathrm{L}$. The mixture was incubated at $25^{\circ} \mathrm{Cfor} 10$ minutes, $16^{\circ} \mathrm{Cfor} 60$ minutes and then $4^{\circ}$ Covernight. GMC plasmids were constructed with the Gibson Assembly kit (New England Biolabs) according to the instructions and $39 \mathrm{ng}$ vector $(3.9 \mathrm{~kb}$ ) was used for the assembly in a total volume of $20 \mu \mathrm{L}$. $2 \mu \mathrm{L}$ ligation mixture was transformed into $50 \mu \mathrm{L} \mathrm{DH} 5 \alpha$ competent cells (transformation efficiency is $>3 \times 10^{8} \mathrm{cfu} / \mu \mathrm{g}$ DNA, Thermo Scientific). The transformant number was counted after overnight incubation. And the positive colony ratio was estimated by PCR characterizations and sequencing validations.

The GFP reporter plasmid (pGFFP) was constructed as described before [9]. An extra LacZ gene flanked by EcoRV/XcmI sites was inserted into the reporter plasmid which would facilitate the clone screening process by applying the blue-white screening. And the Blunt-End ligation or T-A ligation methods could be used thereafter. A 200bp DNA fragment harboring the RGEN target sequences was cloned into the GFP reporter plasmid with T-A clone method.

The golden-gate clone method adapted RGEN vector was constructed by inserting the LacZ gene flanked by BsmBI sites into the BsmBI site of JKJ and gRNA-BL plasmids. The RGEN plasmid construction with Golden-gate method was carried out by mixing $150 \mathrm{ng}$ vector, $1 \mu \mathrm{L}$ annealed oligos, $1 \mu \mathrm{L}$ BsmBI (New England Biolabs), $1 \mu \mathrm{L}$ T4 ligase (New England Biolabs) and $2 \mu \mathrm{L} 10 \mathrm{XT} 4$ ligase buffer (New England Biolabs) in a total volume of $20 \mu \mathrm{L}$. And then the mixture was incubated at $37^{\circ} \mathrm{Cfor} 5$ minutes and $16^{\circ} \mathrm{C}$ for 10 minutes for 10 cycles, followed by $37^{\circ} \mathrm{C} 30$ minutes and $80^{\circ} \mathrm{C} 5$ minutes.

\section{Cell culture}

The human cell line HEK293T was obtained from the American Type Culture Collection (ATCC; Rockville, MD, USA) and cultured in DMEM (GIBCO, Shanghai, China) supplemented with $10 \%$ FBS.

\section{GFP reporter assay}

250ng of gRNA plus 250ng hCas9 plasmid or 500ng cFZ plasmid, 50ng GFP reporter plasmid and 1 $\mu \mathrm{L}$ Lipofactamine2000 (Thermo Scientific) were mixed to transfect 10x104 HEK293T cells/well in p24 plate. The medium was refreshed daily and the cells were analyzed 72 hours post transfection with Flow Cytometery (FC500, Beckman Coulter, Inc.). 


\section{Cellular Physiology Cell Physiol Biochem 2018;48:131-137 \begin{tabular}{l|l} 
and Biochemistry Published onIIne: July 12, 2018 & $\begin{array}{l}\text { DO } 2018 \text { The Author(s). Published by S. Karger AG, Basel } \\
\text { www.karger.com/cpb }\end{array}$ \\
\hline
\end{tabular}}

Xu et al.: Optimized Plasmid Construction Strategy For Cas9

\section{Statistical Analysis}

Data were analyzed by using SPSS software for Windows (SPSS Inc) and shown as means \pm SEM (standard error of the mean). Student $t$-test was used for two-group comparison and one-way ANOVA for multiple group comparisons with normal data distribution, parametric test and Turkey post hoc tests. $P \leq 0.05$ was considered statistically significant.

\section{Results}

To determine which plasmid construction strategy is more cost-effective, time-saving and suitable for high throughput construction with high genome editing efficiency, three different sets of plasmids were compared. The first set (FZ, FZM, cFZ and cFZM) is based on one-plasmid system, which expresses both gRNA and humanized Cas9 (hCas9) in the same vector. The second set (JKJ and BL) uses two-plasmid system. One plasmid contains gRNA while the other plasmid expresses hCas9. The third set also uses two-plasmid system (GMC). However, the GMC uses the Gibson Assemble method for plasmid construction while the second set (JKJ and BL) uses conventional molecular cloning methods of digestion and ligation. Four different target regions of human gene Desmin, which is one of the mutated genes in hereditary cardiomyopathy, were designed with web-based software ZiFiT Targeter [9]. Corresponding modifications were made to adapt to different clone methods (Table 1).

To make the plasmid transformation efficiency comparable, the whole molecular clone procedure was standardized, including the same molar ratio in the vector ligation step, ligation volume (except the Gibson Assemble method) and the same batch of competent cells. Transformant counting showed that the two-plasmid system is much more efficient, normally hundreds of colonies, than the one-plasmid system, only several colonies (Fig. 1A, Table 2). After PCR characterizations and sequencing validations, the positive colony ratio was calculated. Referring to the average positive ratio of PCR characterizations, these systems (except the cFZM) did not show significant difference overall (Fig. 1B). However, the positive ratio of sequencing validation was much higher in the two-plasmid system than in the one-plasmid system $(P<0.05$, Fig. 1B, Table 2$)$.

Interestingly, the large DNA fragment deletion often occurred in the one-plasmid system during plasmid construction (Table 2) according to the sequencing results. The deleted regions

Table 1. Oligos used for gRNA construction

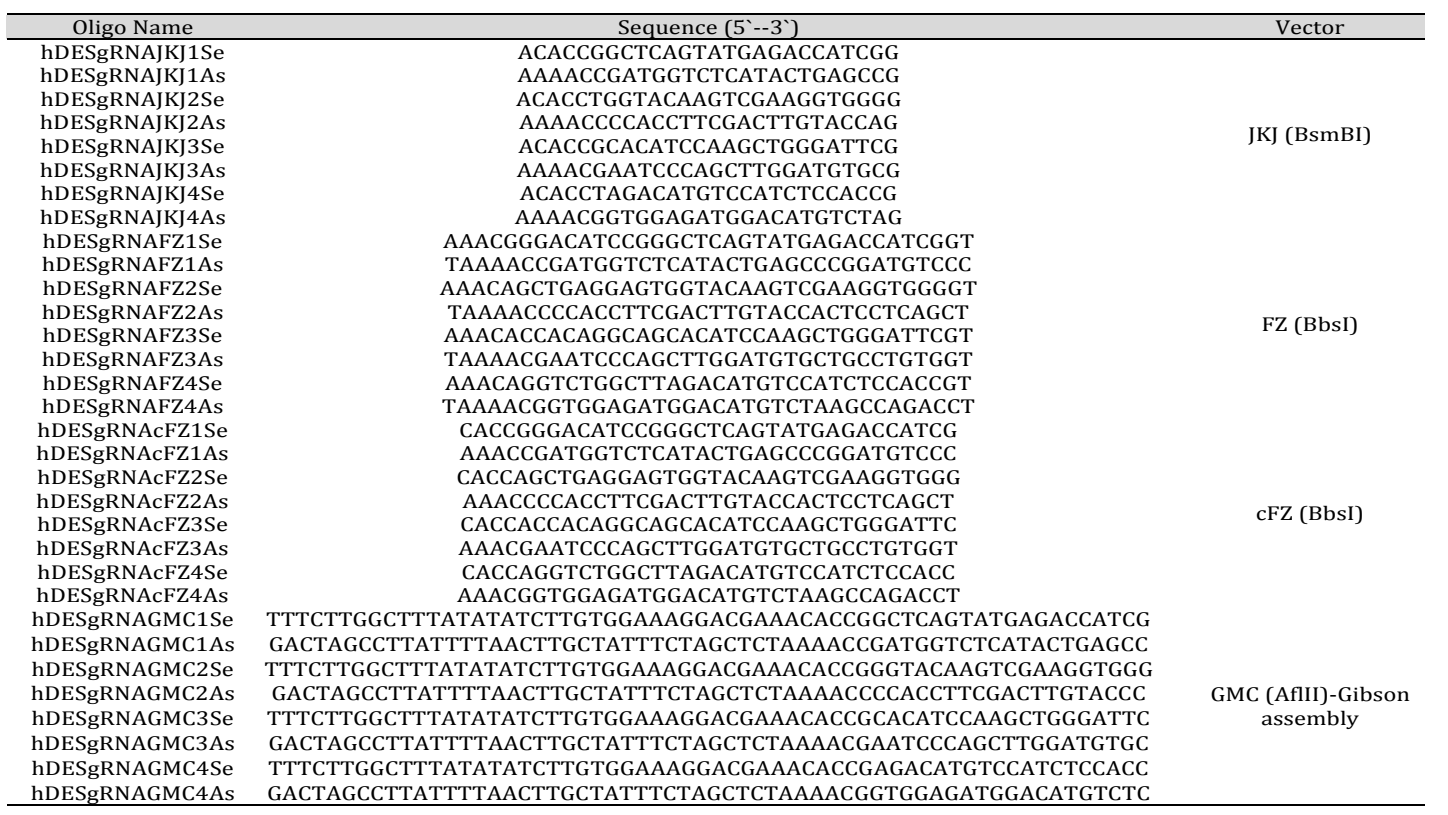


varied, but mostly contained the last DR (direct repeat) and Cas9 (Fig. 2A). This kind of plasmid instability might be caused by DR elements, star activities of BbsI or the gene Cas9. Furthermore, the chimeric form of gRNA without DR elements but containing the Cas 9 also showed large DNA fragment deletion (Fig. 2B, Table 2). And there was no deletion observed in the vector DR-gRNA, which also harbors DR and BbsI sites but without the gene Cas9 (Table 2). Thus, it seems that the gene Cas 9 would induce plasmid instability. However, whether this is true or not needs further investigations. And the plasmid instability could be eliminated when the STBL3 competent cells were used in the plasmid construction procedure (Data not shown). The GMC and JKJ plasmids share the same structure and sequence (both the gRNA and humanized Cas 9 protein sequences). And the Gibson Assembly method (used for GMC), which is more time-consuming and expensive than the conventional digestion-ligation cloning method, did not show any significantly clone efficiency increasement. Thus we used JKJ and BL plasmids in the following experiments. Therefore, during the process of plasmid construction, the twoplasmid system constructed with conventional digestion-ligation molecular cloning method is more efficient.

To measure the differences between the one-plasmid system and two-plasmid system in the genome editing efficiency, a GFP reporter plasmid was applied. The chimeric form of gRNA, in both two-plasmid system and one-plasmid system, produced more GFP positive cells significantly comparing with the reporter plasmid only (Fig. 1C). Furthermore, as demonstrated before [9], the gRNA-BL (the

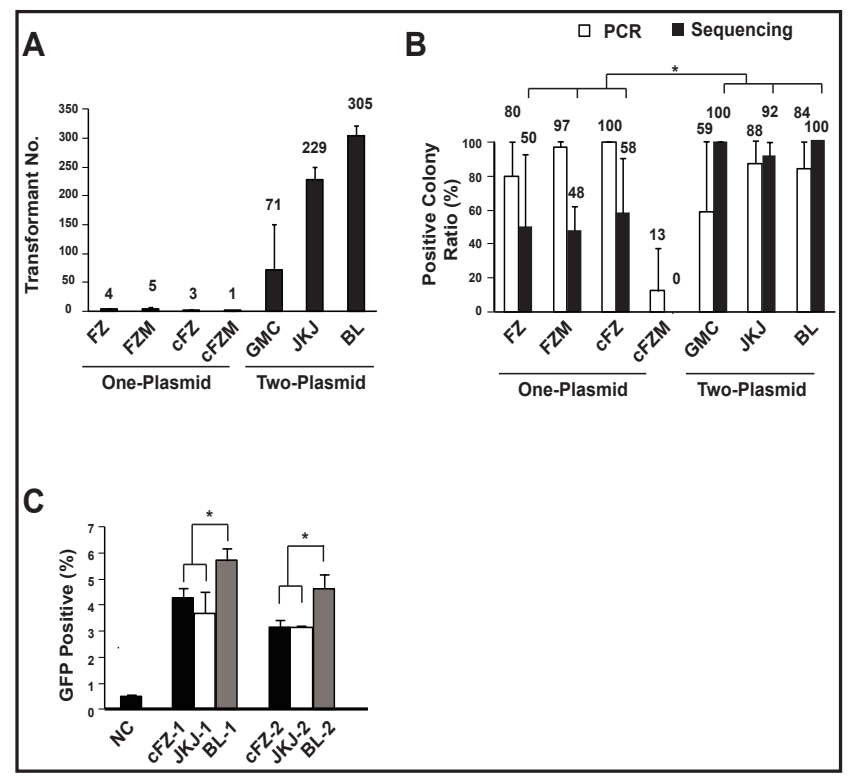

Fig. 1. Plasmid construction strategy comparison. (A) Counting the transformant number after overnight incubation. The number showed the average transformants among the four target regions of human gene Desmin. $N=4$. (B) Positive colony frequency estimated by PCR and sequencing validation. The number showed the average frequency of the positive colonies among the four target regions of human gene Desmin. $\mathrm{N}=4$. * indicates $\mathrm{P}<0.05$. (C) Genome editing efficiency comparison estimated by GFP reporter. Percentage of GFP positive cells via GFP reporter assay when cells transfected with different forms of gRNA (JKJ and BL) plus Cas9 for two-plasmid system or oneplasmid system (cFZ). $\mathrm{N}=3$. * indicates $\mathrm{P}<0.05$. FZ (Addgene 42229), FZM (Addgene 42333), cFZ (Addgene 42230), cFZM (Addgene 42335), GMC (Addgene 41824), JKJ (Addgene 43860), BL: gRNA-BL.

Table 2. Molecular clone and validation results

\begin{tabular}{|c|c|c|c|c|c|}
\hline Plasmid & target site & $\begin{array}{c}\text { Clone No. } \\
\text { (Total/Experiment } \\
\text { repeat) }\end{array}$ & $\begin{array}{l}\text { PCR Characterization } \\
\text { (Positive/total } \\
\text { screened) }\end{array}$ & $\begin{array}{c}\text { Seq validation } \\
\text { (Positive/total) }\end{array}$ & Note \\
\hline \multirow{5}{*}{$\mathrm{JKJ}$} & 1 & $242 / 1$ & & $3 / 3$ & \\
\hline & 2 & $247 / 1$ & $8 / 8$ & $3 / 3$ & \\
\hline & 3 & $227 / 1$ & $6 / 8$ & $3 / 3$ & \\
\hline & 4 & 199/1 & $6 / 8$ & $2 / 3$ & 1bp deletion \\
\hline & 1 & $3 / 1$ & $3 / 3$ & $1 / 3$ & DR and Cas9 deletion \\
\hline \multirow{4}{*}{$\mathrm{FZ}$} & 2 & $2 / 1$ & $2 / 2$ & $2 / 2$ & \\
\hline & 3 & $7 / 1$ & $6 / 7$ & $2 / 3$ & DR and Cas9 deletion \\
\hline & 4 & $6 / 3$ & $2 / 6$ & $0 / 2$ & DR and cas9 deletion \\
\hline & 1 & $10 / 2$ & $10 / 10$ & $5 / 10$ & DR and Cas9 deletion \\
\hline \multirow{4}{*}{ FZM (D10A) } & 2 & $24 / 3$ & $14 / 16$ & $6 / 14$ & DR and Cas9 deletion \\
\hline & 3 & $4 / 1$ & $4 / 4$ & $2 / 3$ & DR and Cas9 deletion \\
\hline & 4 & $4 / 1$ & $4 / 4$ & $1 / 3$ & DR and Cas9 deletion \\
\hline & 1 & $3 / 1$ & $3 / 3$ & $1 / 3$ & Cas9 deletion \\
\hline & 2 & $1 / 1$ & $1 / 1$ & $1 / 1$ & \\
\hline \multirow[t]{2}{*}{ (Chimeric) } & 3 & $3 / 1$ & $3 / 3$ & $2 / 3$ & Cas9 deletion \\
\hline & 4 & $3 / 1$ & $3 / 3$ & $1 / 3$ & Cas9 deletion \\
\hline & 1 & $1 / 1$ & $0 / 1$ & & \\
\hline & 2 & $2 / 1$ & $1 / 2$ & $0 / 1$ & \\
\hline \multirow[t]{2}{*}{ and D10A) } & $\begin{array}{l}3 \\
4\end{array}$ & $0 / 1$ & & & \\
\hline & $\begin{array}{l}4 \\
1\end{array}$ & $\begin{array}{l}0 / 1 \\
10 / 1\end{array}$ & & & \\
\hline \multirow{4}{*}{ GMC } & $\begin{array}{l}1 \\
2\end{array}$ & $\begin{array}{l}10 / 1 \\
9 / 2\end{array}$ & $\begin{array}{l}2 / 0 \\
1 / 9\end{array}$ & $1 / 1$ & \\
\hline & 3 & $173 / 1$ & $8 / 8$ & $3 / 3$ & \\
\hline & 4 & $98 / 1$ & $8 / 8$ & $3 / 3$ & \\
\hline & 1 & $323 / 1$ & $5 / 8$ & $2 / 2$ & \\
\hline \multirow[t]{4}{*}{ BL } & 2 & $293 / 1$ & $7 / 8$ & $2 / 2$ & 1bp deletion \\
\hline & 3 & $315 / 1$ & 8/8 & $2 / 2$ & \\
\hline & 4 & $287 / 1$ & $7 / 8$ & $2 / 2$ & 1bp wrong \\
\hline & & $233 / 1$ & $6 / 8$ & $2 / 2$ & \\
\hline \multirow{2}{*}{ DR-gRNA } & 2 & $275 / 1$ & $8 / 8$ & $1 / 2$ & 1bp deletion \\
\hline & 3 & $\begin{array}{l}315 / 1 \\
254 / 1\end{array}$ & $\begin{array}{l}5 / 8 \\
7 / 8\end{array}$ & $2 / 2$ & 1hpurong \\
\hline
\end{tabular}


new chimeric gRNA structure we identified before) had much higher efficiency than the conventional chimeric structure $(P<0.05$, Fig. 1C).

As the two-plasmid system is much easier to construct and the gRNA-BL shows much higher genome editing efficiency [9], the plasmid construction strategy of gRNA-BL was subjected to further modification. The plasmid construction strategies of both one-plasmid and two-plasmid systems are based on restriction enzyme digestion, gel purification and then ligation (Fig. 3A). The Gibson Assemble method is even more time-consuming which needs one more procedure annealed oligos extension(Fig. $3 \mathrm{~A}$ ). Aiming to reduce the cost, save the time and be suitable for highthroughput RGEN construction, the gRNA-BL expressing vector was modified by inserting the LacZ gene, flanked by two BsmBI recognization sites, into the vector (Fig. 3B). This modified vector is suitable for Golden-gate assembly without the procedures of vector digestion and gel purification. Furthermore, the blue-white screening step increases the ratio of correct colonies. This modified vector could achieve around $100 \%$ positive frequency in both PCR characterizations and sequencing validations. Once the annealed oligos are ready,

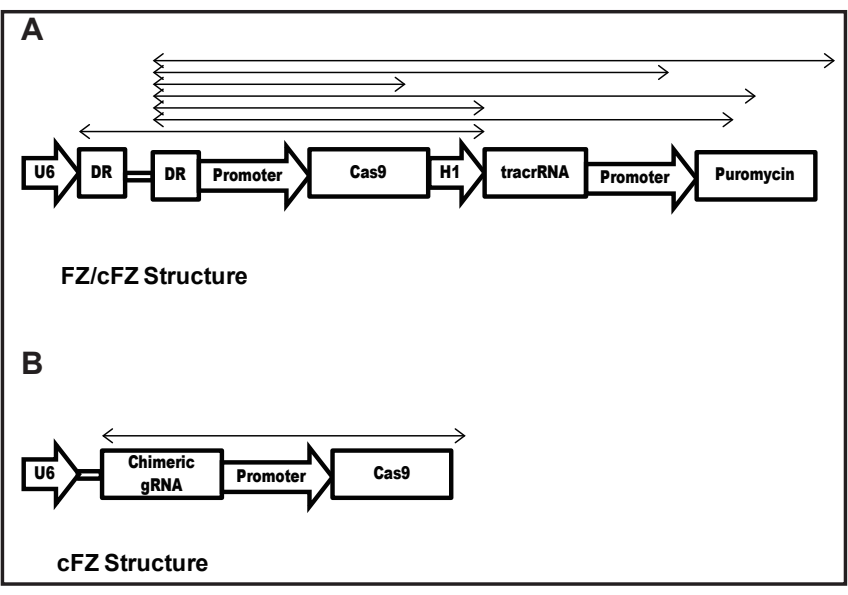

Fig. 2. DNA sequence deleted in the one-plasmid system. Arrows showed the deleted regions.

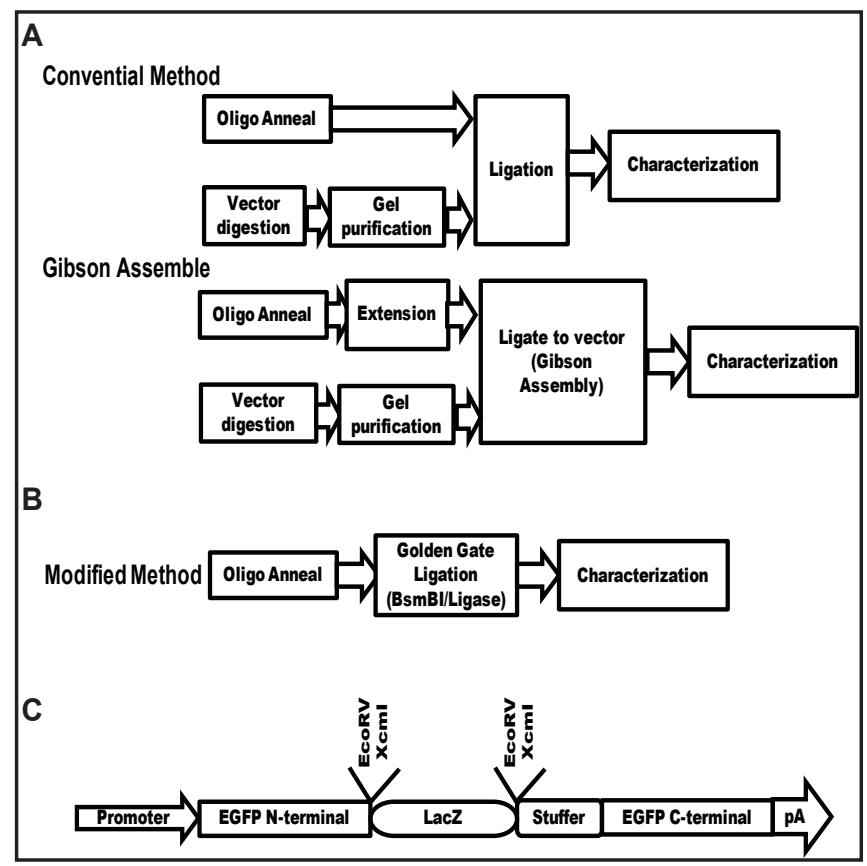

Fig. 3. Flowchart of the conventional molecular cloning procedure (A) and our modified procedures (B and C). the plasmid could be assembled within 3 hours and ready for transformation. In the contrast, the conventional method needs about from 8 to 48 hours. Furthermore, the GFP reporter plasmid was also modified in the similar way (Fig. 3C). The target region could be amplified with PCR and cloned into the reporter vector with either blunt or T-A clone methods.

In summary, the two-plasmid system with our new identified chimeric gRNA is more efficient in both plasmid construction and genome editing. The modified version of gRNA expression vector here would facilitate the process of both plasmid preparation and genome editing efficiency assessment. This is also suitable for high throughput genome editing projects as it is cost-effective and time-saving. 


\section{Discussion}

Short RNA guided endonucleases have paved a new way to manipulate the genome [1, 2]. Although the simple base pairing between the guided RNA of RGEN and the target DNA sequence confers an advantage over protein based DNA sequence recognizations of ZFN and Talen, the gRNA of RGEN also has some tolerance of mismatch pairing, especially in the 5 ' end of the gRNA $[1,4]$. Thus, more efforts should be made to improve the efficiency and specificity. We had identified one new guide RNA structure with higher genome editing efficiency [9]. And we then further have established the cost-effective and time-saving plasmid construction strategy here, which is suitable for the high throughput genome editing.

By following the standardized molecular cloning procedure, the frequency of the correct colonies is much higher in the two-plasmid system than one-plasmid system. Because of the smaller size of the vectors in the two-plasmid system (around 2-3kb) than one-plasmid system $(8-10 \mathrm{~kb})$, the two-plasmid system is much easier to construct. What is more, the vectors in the one-plasmid system are unstable during the molecular cloning process. Therefore, the two-plasmid system expressing our new identified gRNA structure gRNA-BL was subjected to further modifications, including adaption to the Golden-gate clone method and blue-white screening strategy. The modified vector is suitable for high throughput RGEN constructions with reduced cost and time.

The importance of the current study is that we have established one RGEN construction platform with reduced time, cost and labor. Furthermore, we have found that by using the STBL3 component cells, the instability of Cas9 could be eliminated. In the future, more efforts should be made to optimize the Cas9 protein, including discovering new alternative isoforms, testing and reducing their immunogenicity, and establishing the fusion protein with efficient cell membrane permeable abilities. The platform we have established here would facilitate these optimizations a lot with less time and cost.

Although the ribonuceoprotein complexes of recombinant Cas9 protein combined with synthetic guide RNAs have been demonstrated as effective genome editing tool [1214], their efficiency is still much lower than the conventional plasmid transfer approach. Thus, the plasmid based approach would be the mostly used method in the coming years and scientists working on improving the efficiency of custom guide RNA cloning and IVT (in vitro transcription) synthesis will appreciate the strategy employed here.

In conclusion, we have established a cost-effective and time-saving RGEN construction strategy, which is suitable for the high throughput genome editing.

\section{Acknowledgements}

This work was supported by Natural Science Foundation of SZU (2017083), Natural Science Foundation of Shenzhen (JCYJ20170302152735071), and Medical Foundation of Guangdong (A2018308).

\section{Disclosure Statement}

The authors declare no commercial or financial conflict of interests

\section{References}

\footnotetext{
1 Komor AC, Badran AH, Liu DR: CRISPR-Based Technologies for the Manipulation of Eukaryotic Genomes. Cell 2017;168:20-36.

2 Urnov F: Genome editing: The domestication of Cas9. Nature 2016;529:468-469.
} 


\section{Cellular Physiology Cell Physiol Biochem 2018:48:131-137 \begin{tabular}{l|l} 
DOI: 10.1159/000491669 & and Biochemistry \\
Publisned onIIne: July 1Z, 2018 & $\begin{array}{l}\text { 2018 The Author(s). Published by S. Karger AG, Basel } \\
\text { www.karger.com/cpb }\end{array}$
\end{tabular} \\ Xu et al.: Optimized Plasmid Construction Strategy For Cas9}

-3 Tsai SQ, Joung JK: Defining and improving the genome-wide specificities of CRISPR-Cas9 nucleases. Nat Rev Genet 2016;17:300-312.

-4 Shalem 0, Sanjana NE, Zhang F: High-throughput functional genomics using CRISPR-Cas9. Nat Rev Genet 2015;16:299-311.

5 Agudelo D, Duringer A, Bozoyan L, Huard CC, Carter S, Loehr J, Synodinou D, Drouin M, Salsman J, Dellaire G, Laganiere J, Doyon Y: Marker-free coselection for CRISPR-driven genome editing in human cells. Nat Methods 2017;14:615-620.

6 Kim K, Ryu SM, Kim ST, Baek G, Kim D, Lim K, Chung E, Kim S, Kim JS: Highly efficient RNA-guided base editing in mouse embryos. Nat Biotechnol 2017;35:435-437.

7 Gaj T, Gersbach CA, Barbas CF, 3rd: ZFN, TALEN, and CRISPR/Cas-based methods for genome engineering. Trends Biotechnol 2013;31:397-405.

-8 Doench JG, Fusi N, Sullender M, Hegde M, Vaimberg EW, Donovan KF, Smith I, Tothova Z, Wilen C, Orchard R, Virgin HW, Listgarten J, Root DE: Optimized sgRNA design to maximize activity and minimize off-target effects of CRISPR-Cas9. Nat Biotechnol 2016;34:184-191.

-9 Xu J, Lian W, Jia Y, Li L, Huang Z: Optimized guide RNA structure for genome editing via Cas9. Oncotarget 2017;8:94166-94171.

10 Cong L, Ran FA, Cox D, Lin S, Barretto R, Habib N, Hsu PD, Wu X, Jiang W, Marraffini LA, Zhang F: Multiplex genome engineering using CRISPR/Cas systems. Science 2013;339:819-823.

11 Mali P, Yang L, Esvelt KM, Aach J, Guell M, DiCarlo JE, Norville JE, Church GM: RNA-guided human genome engineering via Cas9. Science 2013;339:823-826.

12 Wang L, Shao Y, Guan Y, Li L, Wu L, Chen F, Liu M, Chen H, Ma Y, Ma X, Liu M, Li D: Large genomic fragment deletion and functional gene cassette knock-in via Cas9 protein mediated genome editing in one-cell rodent embryos. Sci Rep 2015;5:17517.

13 Liang X, Potter J, Kumar S, Zou Y, Quintanilla R, Sridharan M, Carte J, Chen W, Roark N, Ranganathan S, Ravinder N, Chesnut JD: Rapid and highly efficient mammalian cell engineering via Cas9 protein transfection. J Biotechnol 2015;208:44-53.

14 Kotani H, Taimatsu K, Ohga R, Ota S, Kawahara A: Efficient Multiple Genome Modifications Induced by the crRNAs, tracrRNA and Cas9 Protein Complex in Zebrafish. PLoS One 2015;10:e0128319. 